\title{
Start-2-End Simulation in FACET Linac
}

\author{
Yipeng Sun, Uli Wienands and Mark Woodley \\ SLAC National Accelerator Laboratory, Menlo Park, California 94025, USA
}

April 1, 2013

\begin{abstract}
Start-2-end simulations are essential to evaluate various beam dynamic aspects. In this note, start-2-end simulation studies are presented for FACET electron linac accelerator, from the damping ring exit point to the final interaction point.
\end{abstract}

\section{Linear optics}

Start-2-end beam dynamics simulation is widely used to optimize the design of modern accelerators. It is also essential in understanding and evaluating various beam dynamics aspects, such as bunch compression, emittance dilution etc..

The energy profile and TWISS parameters of FACET optics Version 31 [1] are shown in Figure 1 below, which are calculated (also designed) by MAD8 [2]. The starting point of this lattice is at the extraction septum of the electron damping ring. It is followed by RTL bunch compressor, a first linac section (LI02-10), a second bunch compressor at LI10, a second linac section (LI11-19), then finally a W-shaped chicane (a third bunch compressor consisting of dipoles, quadrupoles and sextupoles) plus final focus region. The end point of this lattice is the main interaction point (MIP) with plasma wakefield acceleration experiment.

To mitigate the dispersive and chromatic emittance dilution along with a strong linac betatron lattice at lower energy, a gradually increasing staggerd RF phase [3] is adopted in LI02-10. As shown in Figure 1, the electron beam energy is accelerated from $1.2 \mathrm{GeV}$ to $9 \mathrm{GeV}$ in LI02-10, then from $9 \mathrm{GeV}$ to $20.35 \mathrm{GeV}$ in LI11-19.

This FACET MAD8 lattice is then converted into Elegant [4] format, by modifying and applying the FORTRAN code MAD2LTE [5]. The modification of the code is mainly in the RF cavity part, which was previously defined and converted by type (for LCLS case, there are only several types of RF cavity), and currently by actual name and length (each RF cavity is defined seperately with a unique name and length).

In the RTL bunch compressor, there are two quads (QDI454R and QFI464R) with dipole fields, which has no edge effect. All the dipole magnets has FINT=0.5, denoting an average fringing field. Transverse and longitudinal wake fields are employed in all the SLAC S-band RF cavities. Incoherent and coherent synchrotron radiation (ISR and CSR) effects may be included on all the dipole magnets. Meantime CSR could be included for CSR drifts. 
Similarly, the energy profile and TWISS parameters of FACET optics Version 31 are shown in Figure 2 below, which are calculated by Elegant [4]. Observed from a comparison between Figure 1 and Figure 2, also as shown in Table 1, a good agreement is achieved between MAD8 and Elegant calculated energy and TWISS parameters. This is an indication that the conversion from MAD8 to Elegant is successful.

Table 1: Comparison of accelerator parameters calculated by MAD8 and Elegant.

\begin{tabular}{c|c|c}
\hline & MAD8 & Elegant \\
\hline Accelerator length $[\mathrm{m}]$ & 1972.69 & 1972.69 \\
$\beta_{x, I P}[\mathrm{~m}]$ & 0.018 & 0.018 \\
$\beta_{y, I P}[\mathrm{~m}]$ & 0.18 & 0.18 \\
$\alpha_{x, I P}$ & -0.001 & -0.001 \\
$\alpha_{y, I P}$ & 0 & -0.002 \\
$D_{x, I P}[\mathrm{~m}]$ & 0 & 0 \\
$D_{y, I P}[\mathrm{~m}]$ & 0 & 0 \\
$\nu_{x}$ to dump point) & 25.3378 & 25.3362 \\
$\nu_{y}$ (to dump point) & 23.8107 & 23.8087 \\
\hline
\end{tabular}

\section{Start-2-end simulation}

The initial electron beam out of the damping ring is represented by $200 \mathrm{k}$ macro particles. The normalised horizontal and vertical emittance are $33 \mu \mathrm{m}$ and $3.4 \mu \mathrm{m}$ respectively, which are the best guess from measurement. The intial uncorrelated energy spread is $7.9 \times 10^{-4}$ and the RMS bunch length is $5.6 \mathrm{~mm}$. Gaussian distribution is employed for either dimension, with a cutoff at 4 sigma. An asymmetry factor of -0.245 is adopted in generating the longitudinal coordinates, which reflects the potential-well distortion effect in the damping ring.

This electron beam is tracked through the FACET linac, with acceleration, bunch compression and wake fields effects included. The RMS bunch length is compressed by a factor of 5 in the RTL bunch compressor, then a factor of 20 in the sector 10 chicane, and finally a factor of 3 in the $\mathrm{W}$-chicane. The final RMS bunch length is $83 \mu \mathrm{m}$, while the Gaussian fit is $22 \mu \mathrm{m}$. The bunch length and energy spread evolution along the linac are shown in Figure 3.

The transverse emittance is well preserved, and the design transverse beam size is achieved at MIP, as shown in Figure 4. The longitudinal phase space at the entrance and exit of each bunch compressor is shown in Figure 5. Over-compression is employed in a first and second stage bunch compression, while full-compression is adopted in the third stage bunch compression (in W-chicane). The electron beam current profile at the entrance and exit of each bunch compressor is shown in Figure 6 . The beam current is $4 \mathrm{kA}$ out of sector 10 chicane, and it is pushed up to $16 \mathrm{kA}$ after W-chicane. 

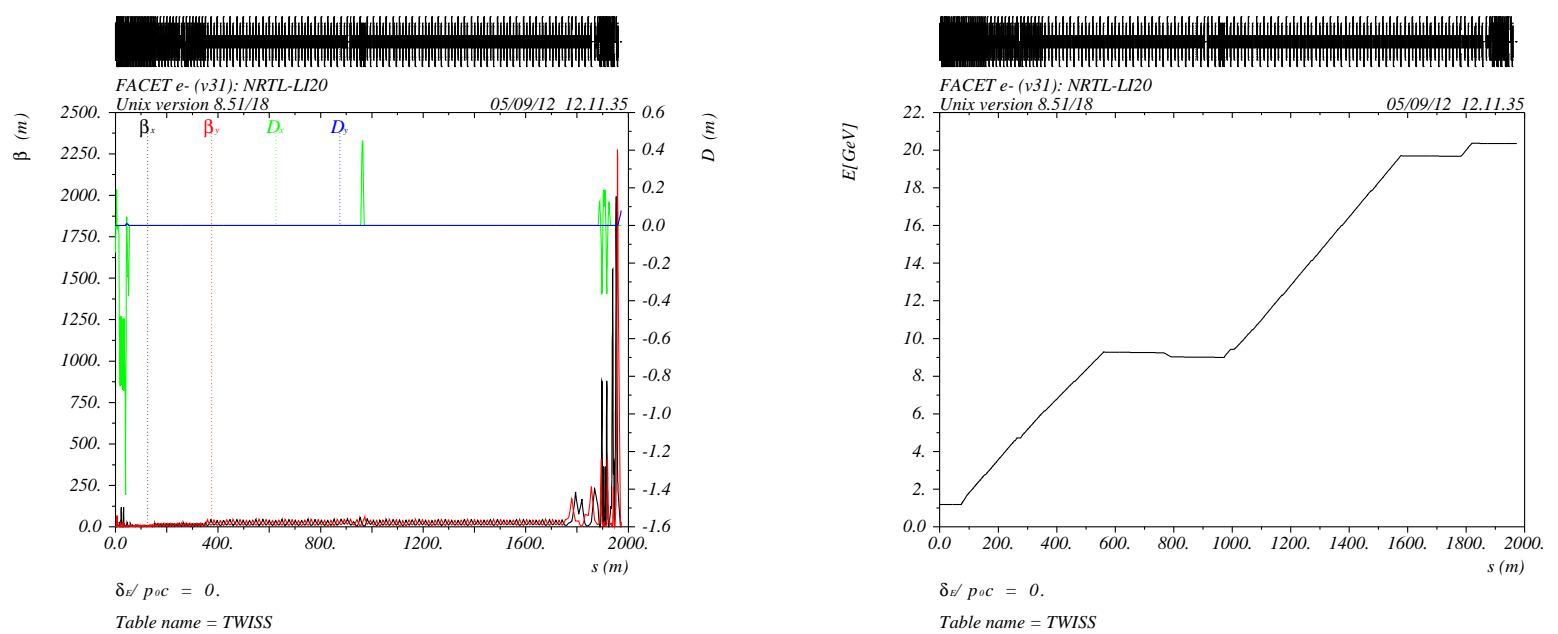

Figure 1: FACET linac TWISS parameters (left) and beam energy (right), calculated from MAD8.

\section{Acknowledgement}

The author would like to thank C. Adolphsen, F.-J. Decker, N. Lipkowitz, Y. Nosochkov, G. Yocky for helpful discussions.

This work was supported by the DOE under Contract DE-AC02-76SF00515.

\section{References}

[1] Mark Woodley etc., FACET optics Version 31 in MAD8.

[2] H. Grote, F.C. Iselin, "The MAD Program (Methodical Accelerator Design) Version 8.15," CERN/SL/90-13 (AP) (1990).

[3] F.-J. Decker etc., SLAC-PUB-15136 (2012).

[4] M. Borland, "elegant: A Flexible SDDS-Compliant Code for Accelerator Simulation," Advanced Photon Source LS-287, (September 2000).

[5] Mark Woodley, MAD2LTE FORTRAN code. 

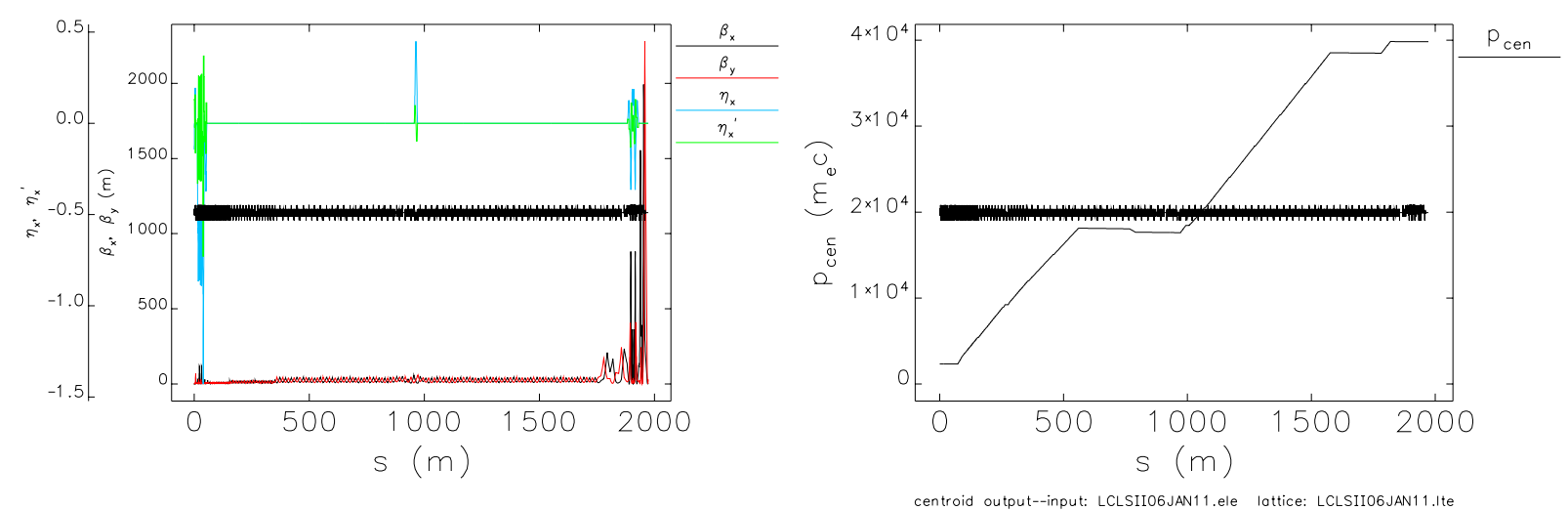

Figure 2: FACET linac TWISS parameters (left) and beam energy (right), calculated from Elegant.

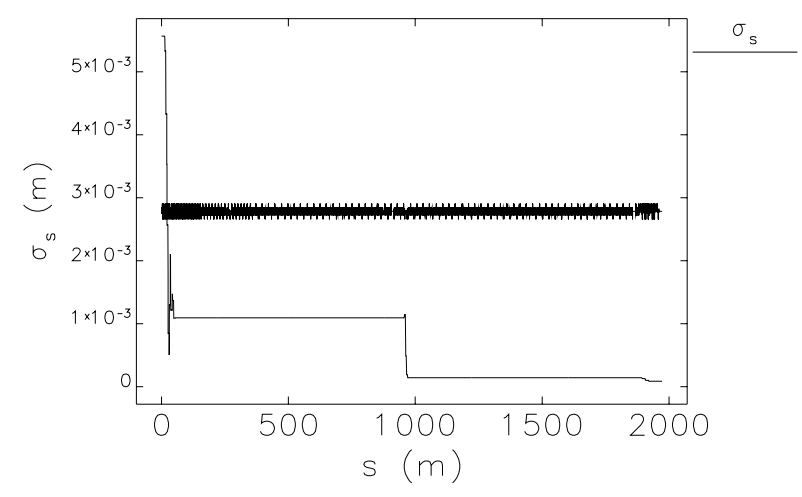

sigma matrix--input: LCLSII06JAN11.ele lattice: LCLSIIO6JAN11. Ite

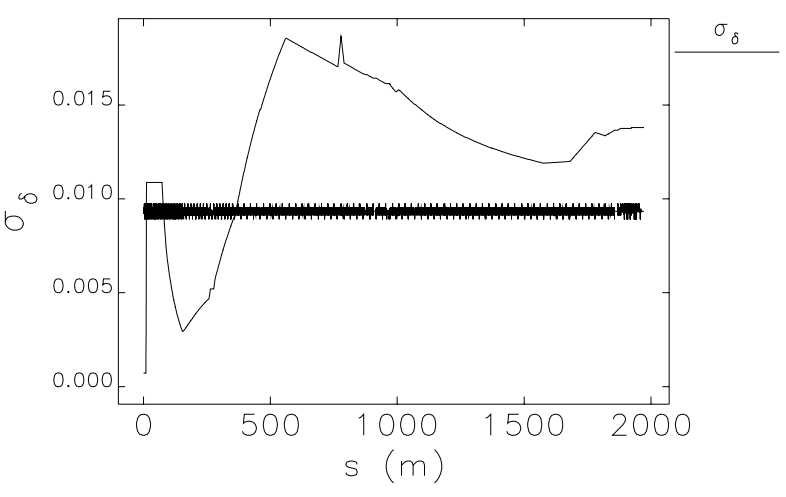

sigma matrix--input: LCLSIIO6JAN11.ele lattice: LCLSIIO6JAN11.Ite

Figure 3: Bunch length (left) and energy spread (right) evolution along FACET linac.

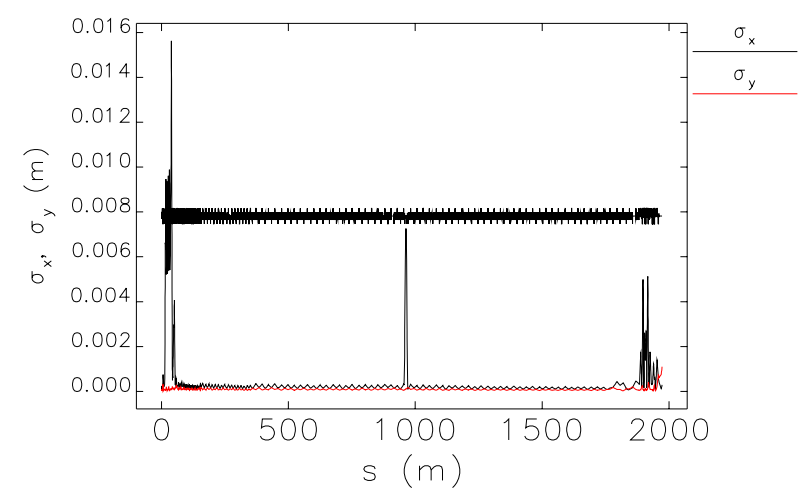

sigma matrix--input: LCLSIIO6JAN11.ele lottice: LCLSIIOGJAN11.Ite

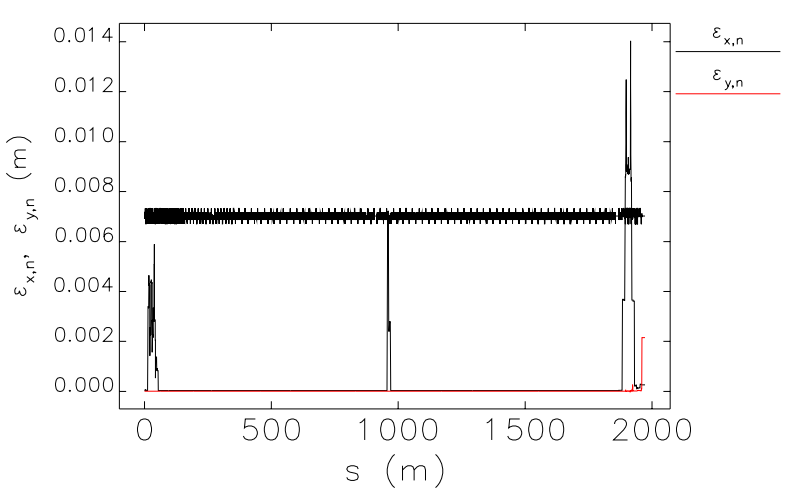

sigma matrix--input: LCLSIIO6JAN11.ele lottice: LCLSIIO6JAN11. Ite

Figure 4: Transverse RMS beam size (left) and projected emittance (right) evolution along FACET linac. 

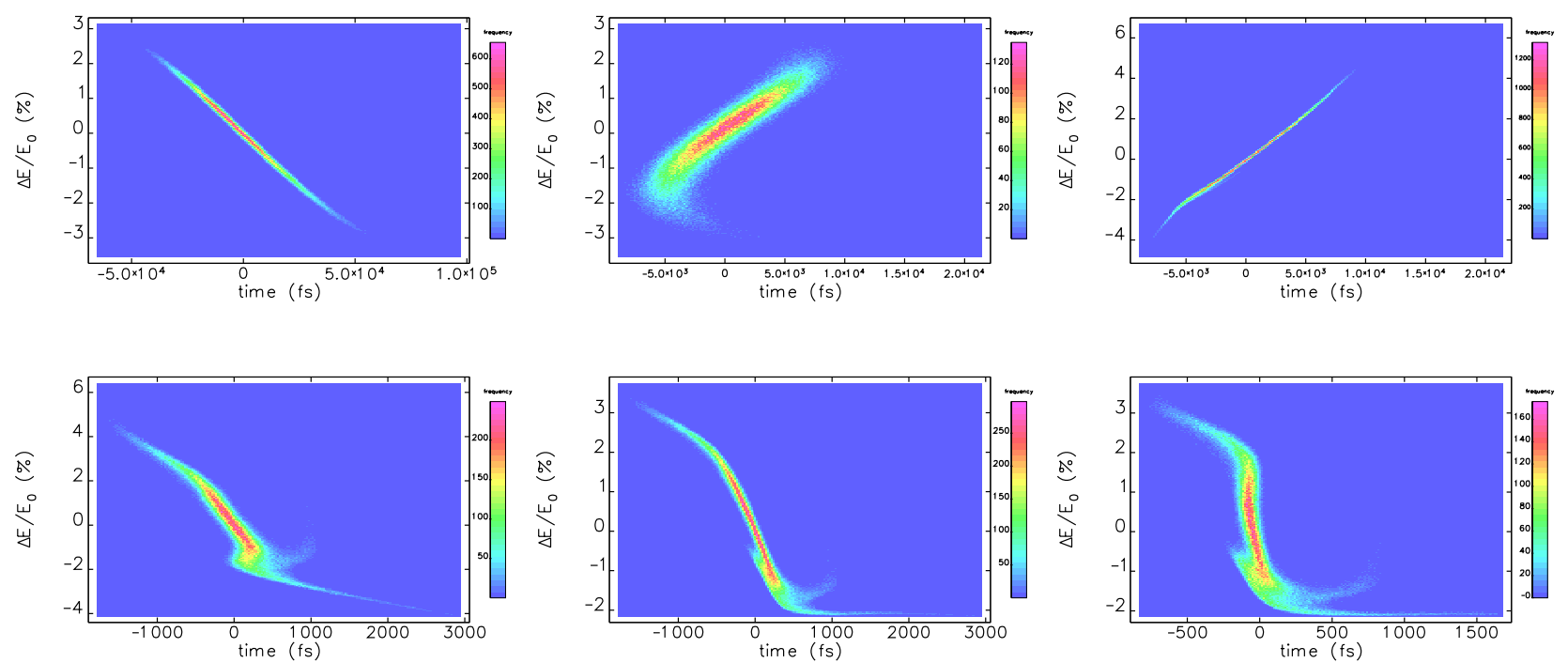

Figure 5: Longitudinal phase space at different locations. From left top to right bottom: RTL bunch compressor entrance; RTL end; sector 10 chicane entrance; sector 10 chicane end; W-chicane entrance; W-chicane end.
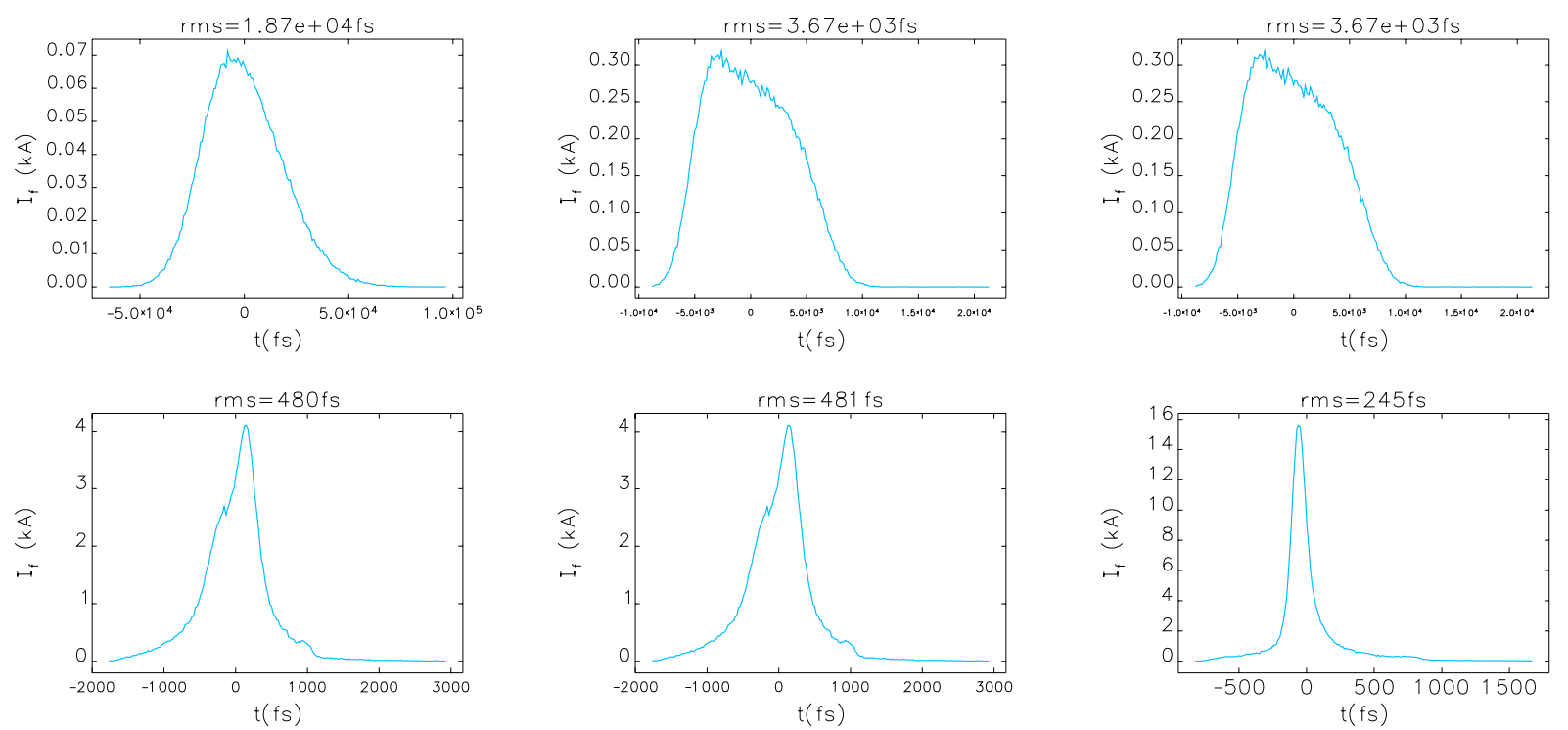

Figure 6: Beam current profile at different locations. From left top to right bottom: RTL bunch compressor entrance; RTL end; sector 10 chicane entrance; sector 10 chicane end; W-chicane entrance; W-chicane end. 